American Journal of Physiology, Vol. 202, No. 4, April 1962

Printed in U.S.A.

\title{
Staircase, rest contractions, and potentiation in the isolated rat heart
}

F. L. MEIJLER

University Department of Cardiology and Clinical Physiology, Wilhelmina-Gasthuis, Amsterdam, The Netherlands 


\title{
Staircase, rest contractions, and potentiation in the isolated rat heart ${ }^{1}$
}

\author{
F. L. MEIJLER \\ University Department of Cardiology and Clinical Physiology, \\ Wilhelmina-Gasthuis, Amsterdam, The Netherlands
}

Meijler, F. L. Staircase, rest contractions, and potentiation in the isolated rat heart. Am. J. Physiol. 202(4): 636-640. I962.Variation in amplitude of isotonic contractions of intact isolated rat hearts, following changes in cycle length, were studied. It was found that a staircase-like phenomenon resembling the original Bowditch effect cannot be evoked in a intact mammalian heart without special measures, such as adding acetylcholine to the perfusion fluid. A steady state relation of rate to amplitude of isotonic contractions was demonstrated. Potentiation of contractility can be originated by sudden changes in stimulation rate. A rest period preceding the changes in stimulation rate does not change the potentiation found originally. At a constant rate the amplitude of a contraction is determined by the preceding cycle length. This relation has been called restitution. Theoretical evidence is presented in an attempt to demonstrate that restitution and potentiation are due to the same process. It can be concluded that Bowditch's staircase does not play a role in the relationship between cycle length and contractility in intact hearts and the statement that restitution and potentiation are due to the same process offers an opportunity to describe all effects of changes in cycle length on isotonic contractions as one phenomenon.

I $1_{T}$ WAS Demonstrated in I87I by Bowditch (I) that contraction height in frog hearts increases from nihil to a certain steady level, when, after a long period of arrest, the heart was stimulated at a constant rate. He called this gradual increase in contraction height "treppe" or "staircase."

Insufficient attention has been paid to the work of Kruta (2) who described a characteristic relationship between stimulation rate and contraction height in mammalian auricle strips. Hajdu (3) and Szent-Gyorgi (4), who made an extensive study of the staircase, classified the relation between frequency and contractility among the staircase phenomenon. At present, even "poststimulation potentiation" is called Bowditch effect (5).

Another important phenomenon demonstrating a

Received for publication $3 \mathrm{r}$ August ig6r.

${ }^{1}$ This study was supported in part by grants from The Netherlands Organization for Pure Research (Z.W.O.), The Hague, The Netherlands. relationship between cycle length and contractility is the potentiating effect of a rest period on subsequent contractions, so-called rest contractions $(6,7)$. This potentiating effect resembles poststimulation potentiation, when a premature beat (8) or contractions with a higher frequency (9) precede the rest period.

In a previous paper (10) the terms staircase, poststimulation potentiation, and rest contraction have been defined. We also indicated that "postextrasystolic potentiation" does not exist as a specific phenomenon (Io). The question arises (I I) whether all these phenomena mentioned above are so closely related that a unitary phenomenologic explanation can be given.

In this study, some experimental and theoretical evidence is given in an attempt to demonstrate a general relationship between cycle length and isotonic contractions of the heart.

\section{MATERIALS AND METHODS}

Isolated hearts from white rats (perfused at $37 \mathrm{C}$ ) were used. Techniques for recording isotonic contractions and electrocardiograms and for complete control of heart rate have been described in the preceding paper (Io).

Special measures are required to evoke Bowditch's staircase phenomenon in the isolated perfused rat hearts. Even in a heart with dissected auricles any rest period (up to $20 \mathrm{sec}$ ) is followed by a ventricular escape showing an increased rest contraction. By adding acetylcholine $\left(\mathrm{IO}^{-6}\right)$ to the perfusion fluid, a cardiac arrest up to 60 sec can be achieved.

Low heart rate can be obtained by dissecting the auricles from the ventricles. Through electrodes, sewed on the area trabecularis of the right ventricle, stimulation rates from I to ro cycles/sec can be applied systematically.

RESULTS

Bowditch's staircase phenomenon has been investigated by means of reapplying stimulation rate $(2-6$ cycles/sec) following rest periods of approximately 60 sec. It was found that after such a period a staircaselike phenomenon can be seen with all stimulation rates investigated ( $2-6$ cycles/sec). 
The steady state relation of rate to amplitude of isotonic contractions has been investigated in a number of hearts.

After dissection of the auricles a short asystolic period

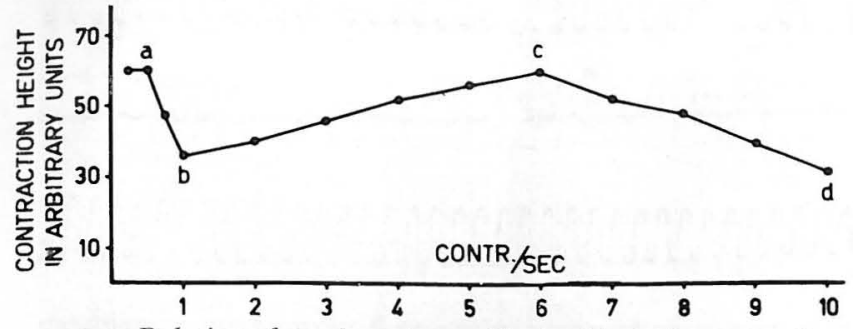

FIG. I. Relation of steady state amplitude of isotonic contractions to stimulation rate. creases until the steady state level belonging to the original frequency of 3 cycles/sec (Figs. I and 2) has been reached. The contraction courses in Fig. $3 A$ are just the reverse from those in Fig. ${ }_{3} C$.

The influence of changes in stimulation rate, following a rest period, on amplitude has been studied. The results are shown in Fig. ${ }_{3} B$ and $D$. In $B$, during a rest period of more than $4 \mathrm{sec}$, the original driving frequency of 3 cycles/sec has been increased to 5 cycles/sec. In $D$, during a rest period of more than $3 \mathrm{sec}$, the reverse has been done (from 5 to 3 cycles/sec). As a matter of fact, the first contraction occurring after the rest period is a rest contraction. But, after the rest contraction the pattern of the change in contractions in Fig. ${ }_{3} B$ is equal to
(5-10 sec) ends with a maximal large contraction, followed by approximately ten beats of equal size with a cycle length of $2-3 \mathrm{sec}$ (Fig. ia). Hereafter, the beats have a cycle length of approximately I.5 sec (idioventricular rhythm). The amplitude of these contractions is smaller than the preceding slower beats.

The influence of driving the heart with frequencies varying from I to Io cycles/ sec on steady state contraction height are given in Fig. 2.

A sudden change in rate gives rise to a characteristic pattern in course of isotonic contractions during transition time before reaching steady state. The results of a representative experiment are shown in Fig. 3 .

In Fig. $3^{A}$ driving frequency has been increased from 3 to 5 cycles $/ \mathrm{sec}$. After an initial decrease, contraction height increases until the constant amplitude belonging to that frequency (Figs. I and 2) has been reached.

In Fig. ${ }_{3} C$ the reverse has been done, decreasing driving frequency from 5 to 3 cycles/sec. The first contractions of the 3 cycles/secrhythm are enlarged (poststimulation potentiation) and contraction height de-

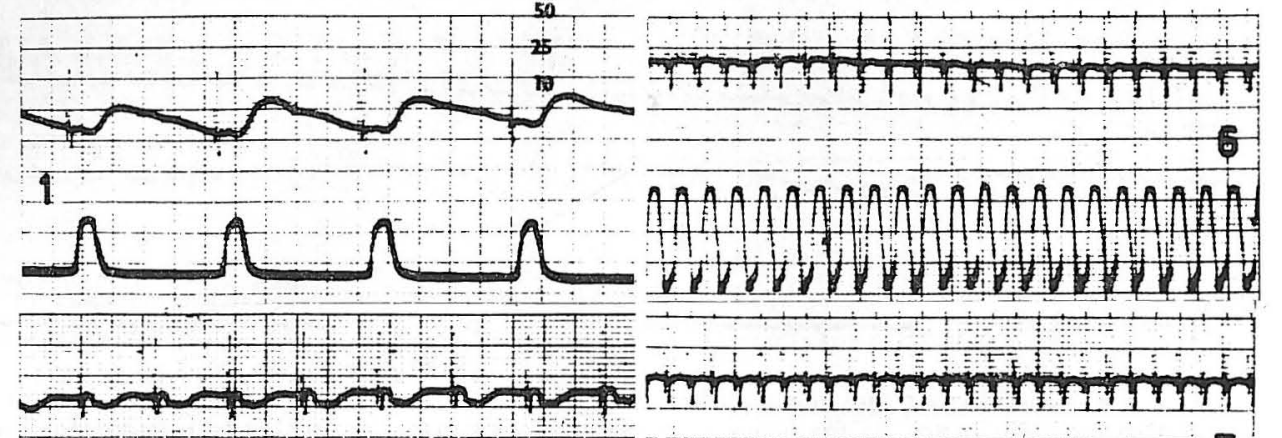

$2+1+1+1+1+1$
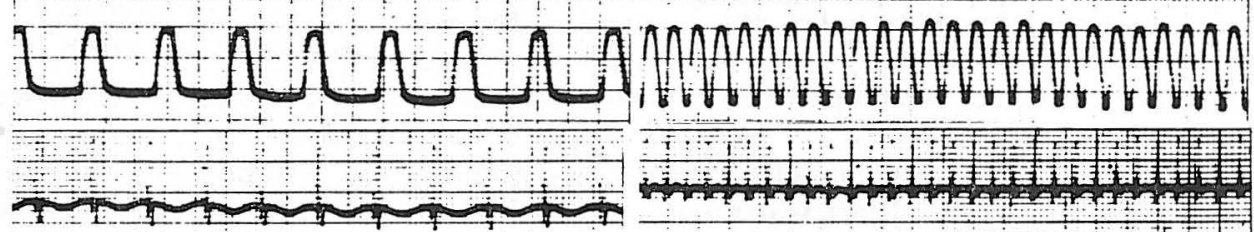

3

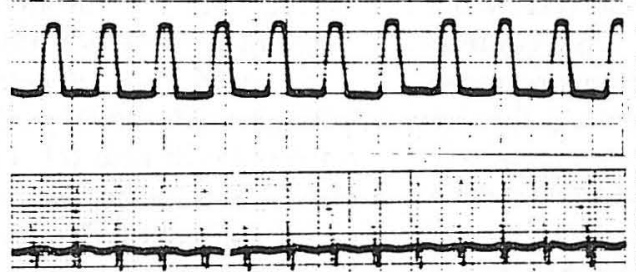

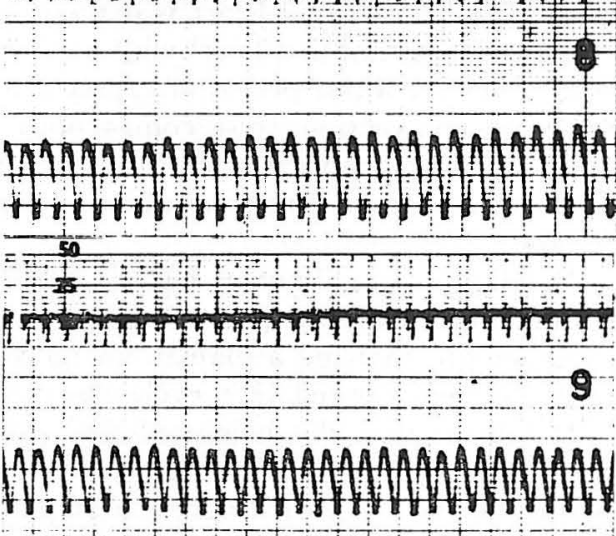

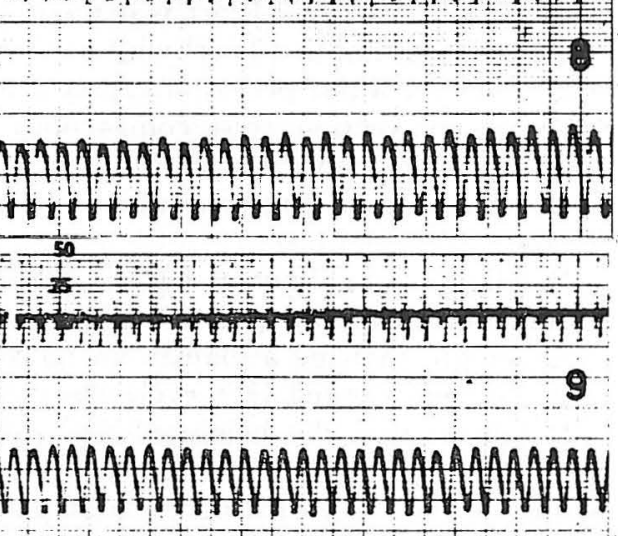

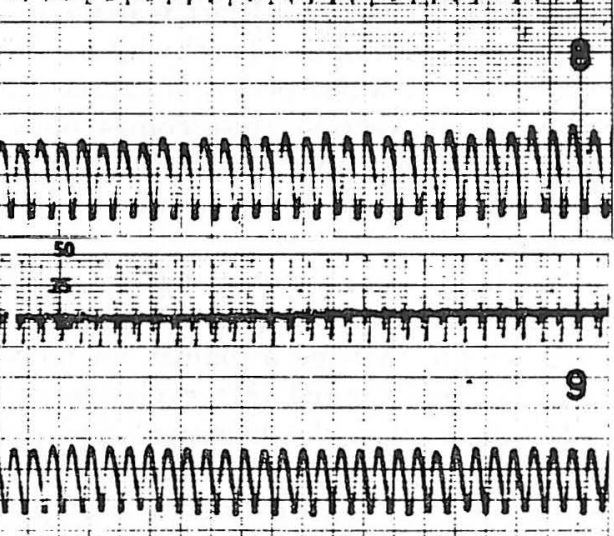

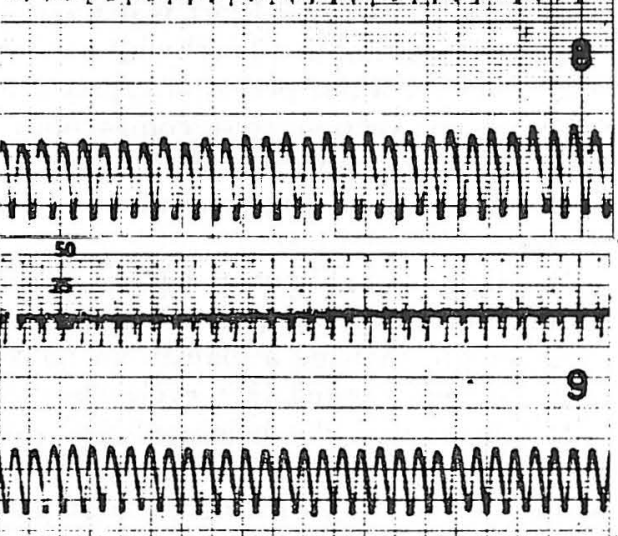
द IीAीAीAीAीAीAA

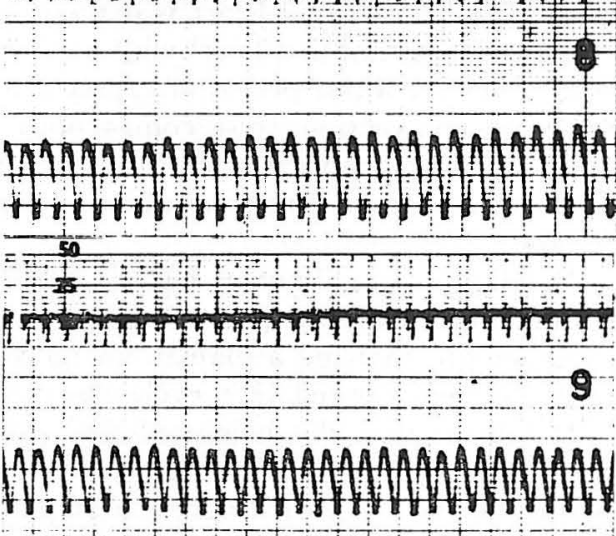

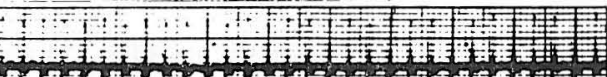

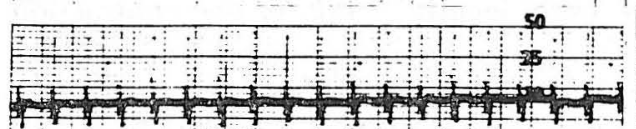
E 5

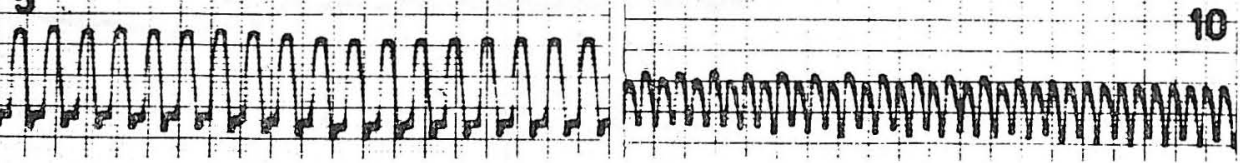

FIG. 2. Electrocardiogram and steady state amplitude of contractions of an isolated perfused heart. Driving frequency is increased from I cycle/sec in I until io cycles/sec in Io. Note optimal contractility at 6 cycles/sec and increasing alternation of contractions at still higher driving frequencies. Paper speed, $25 \mathrm{~mm} / \mathrm{sec}$. 


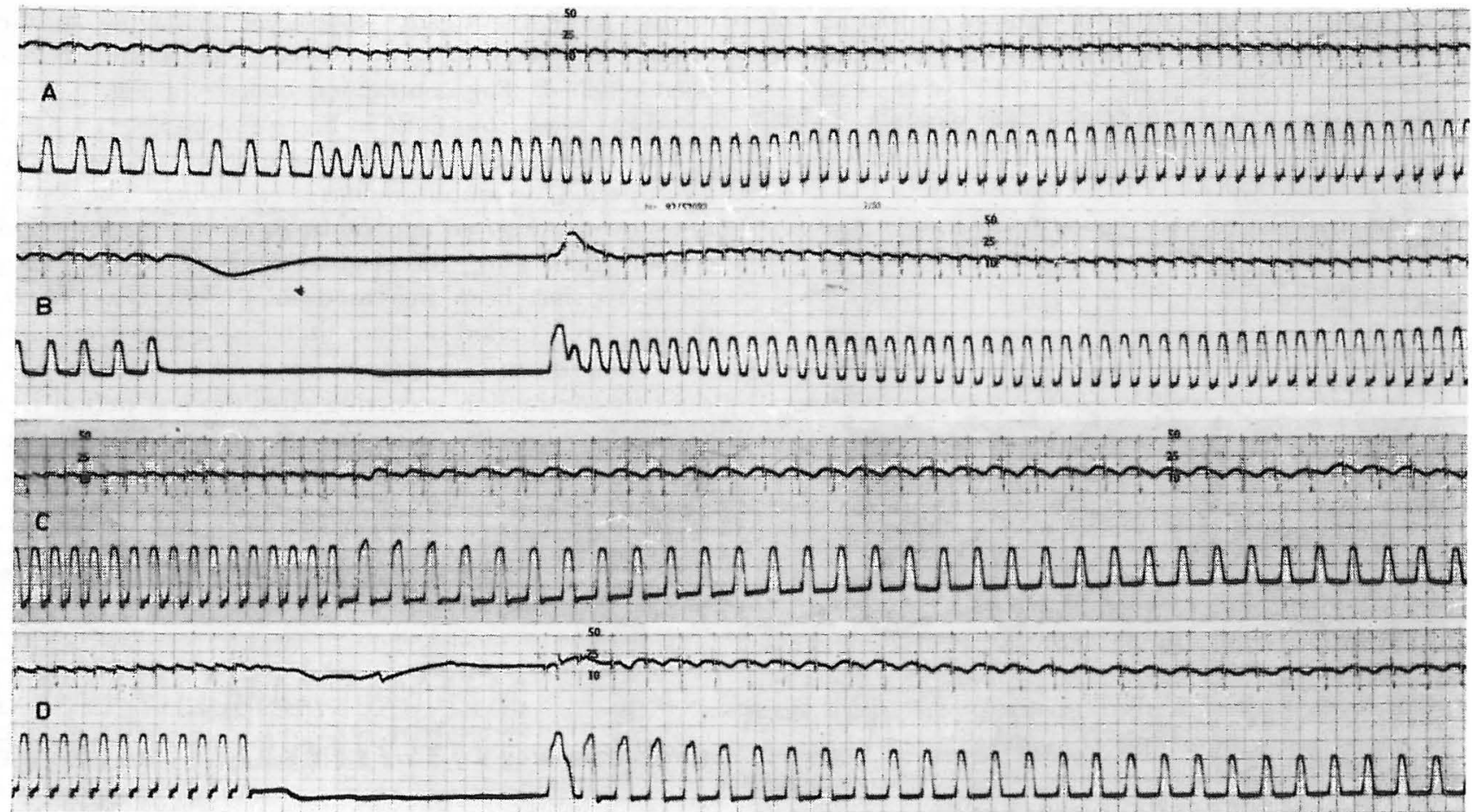

FIG. 3. Electrocardiogram and apical displacement record of an isolated perfused rat heart. $A$ : frequency is increased from 3 to 5 cycles/sec; $B$ : as $A$, but after a rest period; $C$ : frequency decreased from 5 to 3 cycles/sec. $D:$ as $C$, but after a rest period. The rest

that in Fig. $3 A$; the same applies for the contractions in Fig. $3 D$ and $C$.

An example of the influence of a rest period on isotonic contractions in a rat's heart is demonstrated in Fig. 4. It can be seen that in a heart with basal stimulation rate of 4 cycles/sec a rest period of $\mathrm{I} .3 \mathrm{sec}$ is followed by one enlarged contraction (rest contraction). The contractions following the increased rest contraction are somewhat smaller than the contractions preceding the rest period.

In all our experiments the rest contraction reaches its maximum after a period of approximately $\mathrm{I} .5 \times$ cycle length, showing a plateau for more than $20 \mathrm{sec}$ at each frequency tested ( $2-6$ cycles/sec).

It follows that the height of the rest contraction is determined by the height of the contractions belonging to the frequency preceding the rest period, presuming that the rest period is longer than $1.5 \times$ cycle length period is followed by a rest contraction. Note similarity of time course of contractility in $A$ and $B$ and in $C$ and $D$. Paper speed, $25 \mathrm{~mm} / \mathrm{sec}$.

and shorter than $20 \mathrm{sec}$. This is demonstrated in Fig. $3 B$ and $D$.

A time course of myocardial contractility resembling poststimulation potentiation can occur under a variety of experimental conditions. This is demonstrated in Fig. 5, which is the diagrammatic representation of an experiment in which the influence of duration of a high stimulation rate preceding a rest period was studied.

Figure $5^{A}$ shows poststimulation potentiation during a decrease of rate (cf. Fig. $5^{A}$ with Fig. ${ }_{3} C$ ). Figure ${ }_{5} B$ also shows poststimulation potentiation during a decreased rate, but after a rest period and a rest contraction (dotted line) (cf. Fig. $5^{B}$ with Fig. $3^{D}$ ). Figure ${ }_{5} C$ is the same as Fig. $5 B$, but with a shorter period of the high stimulation rate. The same applies for Fig. ${ }_{5} D$, but with a still shorter period of high driving frequency. Finally, in Fig. $5 E$ the increased rate period has a duration of one contraction (premature beat), and now the

FIG. 4. Electrocardiogram and apical displacement record, showing rest contraction of an isolated rat heart. Notice the slight decrease of amplitude of the first contractions, following the rest contraction. Stimulation rate, 4 cycles/sec. Duration rest period, I.3 sec. Paper speed, $25 \mathrm{~mm} / \mathrm{sec}$

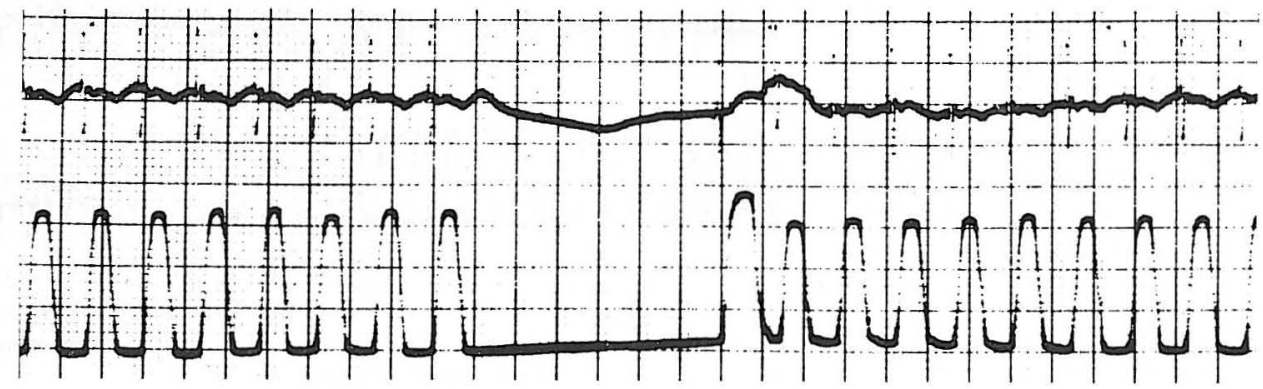




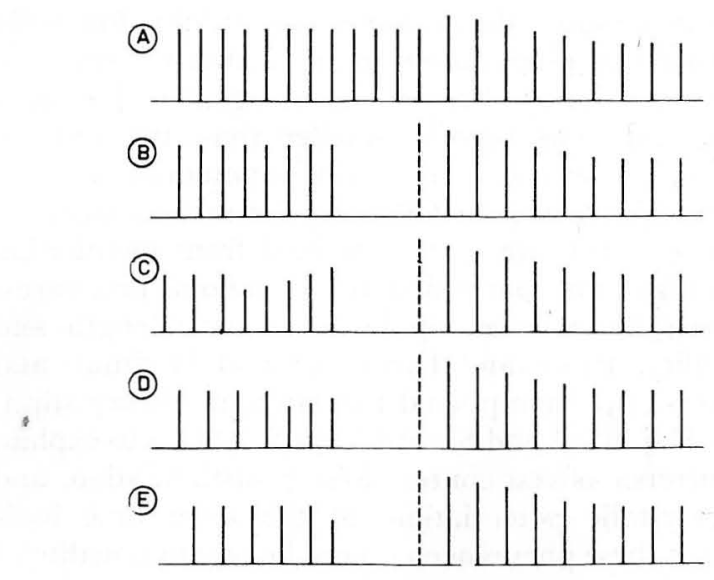

FIG. 5. Diagrammatic representation of: $A$ : poststimulation potentiation. Compare this diagram with Fig. $3 C$. B: poststimulation potentiation occurring after a rest period and a rest contraction (broken line). Compare this diagram with Fig. ${ }_{3} D$. C. as $B$, but with a shorter duration of high stimulation rate. $D$ : as $C$, with a still shorter duration of high stimulation rate. $E$ : as $C$ and $D$. The increased rate has a duration of one contraction (premature beat).

rest period exceeding the compensatory pause is also followed by (poststimulation) potentiation.

\section{DISCUSSION}

Until now, Bowditch's staircase phenomenon (I) has only been described in amphibian hearts or in strips of mammalian myocardium. It was demonstrated that in the isolated perfused rat heart only by addition of acetylcholine to the perfusion fluid can the duration of the rest period be increased sufficiently long to get a staircase phenomenon resembling the original Bowditch effect. Since acetylcholine suppresses mechanical activity of the heart directly, this method might not be reliable. The question arises whether the staircase phenomenon plays any role at all in the relationship between cycle length and contractility in intact mammalian hearts.

It is of importance to note the existence of a characteristic relationship between rate and steady state ampli-

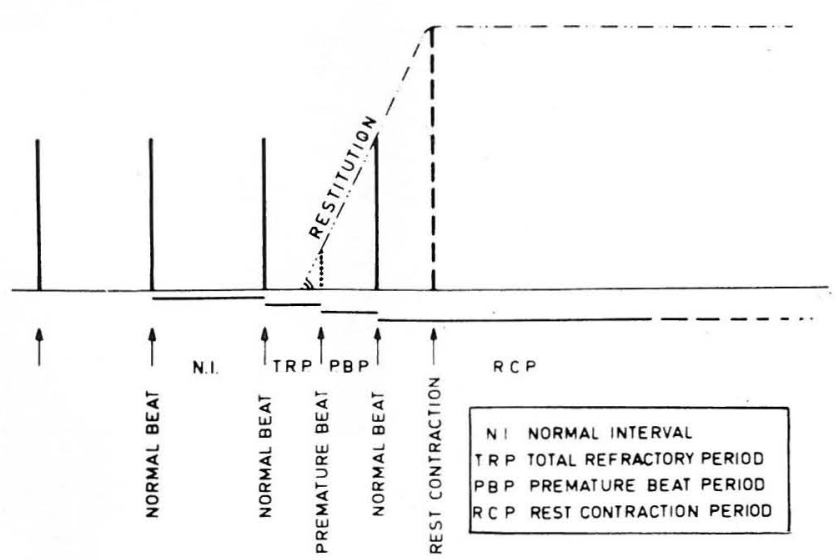

FIG. 6. Schematic representation of the relationship between cycle length and contraction height (restitution).

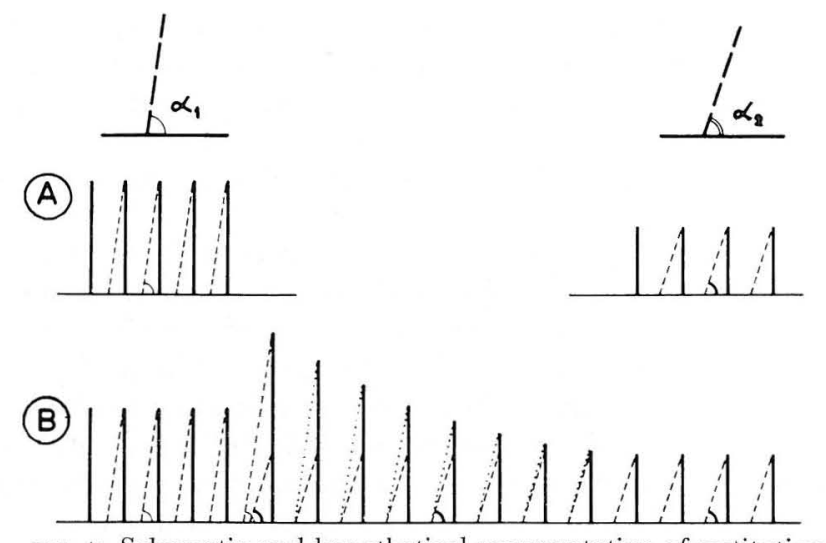

FIG. 7. Schematic and hypothetical representation of restitution and potentiation process. A: restitution (broken line) at a high rate $\left(\alpha_{1}\right)$ and at a low rate $\left(\alpha_{2}\right)$ in the steady state. $B:$ poststimulation potentiation is the discrepancy between restitution observed (dotted line) and restitution belonging to the new frequency in the steady state $\left(\alpha_{2}\right)$. There is no direct evidence about the moment at which restitution starts. More or less arbitrarily we have taken half of the duration of the cardiac cycle representing approximately the end of the refractory period.

tude of contractions $(2,12)$. We demonstrated that in an isolated rat heart perfused at $37 \mathrm{C}$ the steady state contraction height is an almost linear function of stimulation rate between $\mathrm{I}$ and 6 cycles/sec (Fig. I $b-c$ ). At a certain constant rate the amplitude of a contraction is determined by the duration of the cycle length preceding it. This cycle length determines whether a contraction is a premature beat, a normal contraction, or a rest contraction. Amplitude of contraction as a function of the preceding cycle length has been called "restitution" of contractility (13).

Derived from the papers of Kruta and Braveny (I3), Siebens et al. (I 4 ), and our own observations, a schematic illustration of restitution is presented in Fig. 6. To a certain limit, restitution of contractility is an almost linear function of time. The tangent of the angle $\alpha$ formed by the line representing this function and the abscissa offers a quantitative indication of the velocity of the restitution process (Figs. 6, 7). In the steady state tangent $\alpha$ is constant at a constant regular rate. It follows that from I to 6 cycles/sec (Fig. I) a higher frequency is related to a larger tangent $\alpha$.

When driving frequency is suddenly changed (Fig. $3^{A}$ and $C$ ), it takes time before the contractions reach the height belonging to their new frequency in the steady state. It can as well be said that it takes time before the velocity of the restitution process, i.e., tangent $\alpha$, reaches the value belonging to the new frequency. Potentiation can be defined as a discrepancy between tangent $\alpha$ at that moment and tangent $\alpha$ belonging to the introduced frequency in the steady state (Fig. 7), potentiation being positive (poststimulation potentiation, Figs. ${ }_{3} C$ and ${ }_{7} B$ ) if tangent $\alpha_{1}$ is larger than the tangent $\alpha_{2}$ belonging to the new frequency in the steady state, and potentiation being negative (at the beginning of an increase in frequency, Fig. $3^{A}$ ) if tangent $\alpha_{1}$ is smaller than the tangent $\alpha_{2}$ belonging to the new frequency. 
It is indicated in Fig. 6 that restitution reaches its maximum after approximately $1.5 \times$ cycle length and stays constant for at least $20 \mathrm{sec}$ in our experiments, keeping rest contractions constant at their maximum from approximately I.5 $\times$ cycle length to at least $20 \mathrm{sec}$.

From Fig. $3 B$ and $D$ we must accept the fact that during a long interruption the heart goes on or stays in a metabolic condition which has been originated by the foregoing frequency. We also demonstrated (Fig. 5) that the duration of increased stimulation rate before the rest period has no appreciable influence on the course of contractions coming thereafter. Thus the last cycle length preceding the rest period has, relatively, the greatest influence on the contractions following this period. One shorter cycle length suffices to change postrest potentiation pattern.

Figure 4 demonstrates that the first contractions after the rest period, having the same rate as the contractions before the rest period, have become somewhat smaller.

\section{REFERENCES}

. Bowditch, H. P. Arb. Physiol., Leipzig 6: I39, I871.

Kruta, V. Arch. intern. physiol. 45:332, I937.

. Hajdu, S. Am. J. Physiol. i 74: 371, 1953.

4. Szent-Gyorgyi, A. Chemical Physiology of Contraction in Body and Heart Muscle. New York: Academic Press, I953.

5. Sarnoff, S. J., and J. H. Mitghell. Am. J. Med. $30: 747$, I 96 I.

6. Rosin, H., and A. Farah. Am. J. Physiol. i 8o: 75, 1955.

7. Cattell, McK., and H. Gold. Am. J. Physiol. i82: 307 , i 955.

8. Garb, S., and M. Penna. Am. J. Physiol. i 82:6o i, 1955.

9. Speirs, R. L. Nature i $84: 66$, 1959.

io. Meijler, F. L., F. v. D. Bogaard, L. H. v. D. Tweel, and D. Durrer. Am. J. Physiol. 202 : 63i, ig62.
This phenomenon is the reverse of potentiation following an interpolating premature beat (I0). After the rest contraction the velocity of restitution (tangent $\alpha$ ) has been decreased and has become smaller than the original tangent $\alpha$, giving rise to a "negative potentiation" and decreasing the first beats following the rest contraction.

In these papers we have abstained from mentioning the investigations concerning the metabolic process(es) underlying the relationship between cycle length and contractility. Rosin and Farah (6) and Hoffman and co-workers ( 15 ) have proved that an ionic theory stipulated by Hajdu (3) and Szent-Gyorgyi (4) fails to explain the occurrence of rest contractions, poststimulation, and postextrasystolic potentiation, at the same time indicating that these phenomena do not belong to Bowditch's staircase phenomenon.

The influence of possible catecholamine stimulus on the relationship between cycle length and isotonic contraction in the isolated rat heart will be dealt with in a subsequent paper (I6).

i i. Mommaerts, W. F. H. M., B. C. Abbott, and W. J. Whalen. Structure and Function of Muscle. New York: Academic Press, I g6o, vol. II, p. 5 I 7 .

12. Katzung, B., H. Rosin, and F. Sahneider. J. Pharmacol. Exptl. Therap. 120: 324, 1957.

13. Kruta, V., and P. Braveny. Nature i 87:327, ig6o.

i 4. Siebens, A. A., B. F. Hoffman, P. F. Cranefield, and C. McC. Brooks. Am. J. Physiol. I $97: 97$ I, 1959.

15. Hoffman, B. F., E. Bindler, and E. E. Sugkling. Am. J. Physiol. 1 85: 95, 1956.

i6. Meijler, F. L., And D. Durrer. Am. J. Physiol. In press. 